Check for updates

Cite this: RSC Adv., 2018, 8, 38860

\title{
Halogeno-substituted indazoles against copper corrosion in industrial pickling process: a combined electrochemical, morphological and theoretical approach $\dagger$
}

\author{
Yujie Qiang, ${ }^{a}$ Shengtao Zhang, (D) *a Qin Xiang, (DD ${ }^{a}$ Bochuan Tan, ${ }^{a}$ Wenpo Li, ${ }^{\text {a }}$ \\ Shijin Chen ${ }^{\mathrm{b}}$ and Lei Guo (D) ${ }^{\mathrm{c}}$
}

\begin{abstract}
The inhibitive properties of four indazole-based compounds (IA, 4-FIA, 4-CIA, and 4-BIA) on copper corrosion in $0.5 \mathrm{M} \mathrm{H}_{2} \mathrm{SO}_{4}$ solution were investigated using electrochemical measurements, surface characterization techniques and molecular modelling methods. Electrochemical tests indicate that the inhibition efficiencies increase with incremental concentration and all halogeno-substituted indazoles (HIAs) possess superior inhibitive ability to native IA. The specific rating of inhibition performance obeys the order: $\mathrm{IA}<4-\mathrm{FIA}<4-\mathrm{BIA}<4-\mathrm{CIA}$. All inhibition efficiencies of HIAs obtained were over $96 \%$ in $1 \mathrm{mM}$, especially, 4-CIA reaches $99.6 \%$. Moreover, the corresponding inhibition mechanism was elucidated via quantum chemical calculations allied to molecular dynamics simulation. In summary, the present study can help us to gain insight into the effect of halogeno-substitution on the inhibition efficiency of the IA molecule.
\end{abstract}

Received 5th October 2018

Accepted 12th November 2018

DOI: $10.1039 / \mathrm{c} 8 \mathrm{ra} 08238 \mathrm{c}$

rsc.li/rsc-advances
The inhibition behaviour of isatin on copper corrosion in sulfuric acid was investigated by Quartarone et $a l .{ }^{18}$ The inhibition capacity of rhodanine- $N$-acetic acid on corrosion of copper in $0.5 \mathrm{M} \mathrm{H}_{2} \mathrm{SO}_{4}$ medium was studied by using electrochemical methods and morphology observation. ${ }^{19}$ Even so, the main method of exploring inhibitors is still blind filtration nowadays. The complicated and indistinct inhibition mechanism is the major obstacle in designing new organic compounds as corrosion inhibitors.

To alleviate the above problem, molecular dynamics (MD) simulation allied to quantum chemical calculation can be combined to get insight into the inhibition mechanism of organic inhibitor on metal surface at the molecular and atomic level. These computational methods are widely reported as a powerful tool in molecular design of corrosion inhibitor. ${ }^{20-25}$ The corrosion inhibition mechanism of three triazole derivatives for copper in acid system was investigated by quantum chemical and molecular dynamics approaches by our group. ${ }^{26}$ The results obtained reveals that the energies of $\sigma$-molecular orbitals have a remarkable influence on the adsorption performance of corrosion inhibitor. Wang et al. systematically evaluated the inhibitive properties of two quinoline derivatives namely, 8-nitroquinoline and 8-aminoquinoline, for AA5052 aluminum corrosion using electrochemical experiments in combination with density functional theory (DFT). ${ }^{27}$ The calculated results indicate that a strong hybridization happens between sp-orbital of $\mathrm{Al}$ atoms and p-orbital of the inhibitors. Kokalj et al. studied the binding roles of mercapto, benzene,
${ }^{a}$ School of Chemistry and Chemical Engineering, Chongqing University, Chongqing 400044, China. E-mail: stzhang_cqu@163.com; Tel: +86 2365678934

${ }^{b}$ Bomin Electronics Ltd., Meizhou 514021, China

'School of Materials and Chemical Engineering, Tongren University, Tongren 554300, China

$\dagger$ Electronic supplementary information (ESI) available. See DOI: $10.1039 / \mathrm{c} 8 \mathrm{ra} 08238 \mathrm{c}$ 
<smiles>c1ccc2[nH]ncc2c1</smiles><smiles>Fc1cccc2[nH]ncc12</smiles><smiles>Clc1cccc2[nH]ncc12</smiles><smiles>Brc1cccc2[nH]ncc12</smiles><smiles></smiles>

IA<smiles>Fc1cccc2[nH][nH+]cc12</smiles>

4-FIA<smiles></smiles>

4-CIA<smiles>Brc1cccc2[nH][nH+]cc12</smiles>

4-BIA

Fig. 1 Lewis structures of IA, 4-FIA, 4-CIA, and 4-BIA molecules in neutral (Mol, top row), and protonated cationic (MolH ${ }^{+}$, bottom row) forms.

and methyl groups of imidazoles on copper surface in the corrosion inhibition process by DFT calculation. ${ }^{28}$ The results well captured the inhibition efficiency trend obtained experimentally, and testify mercapto-substituted imidazoles bond stronger to the copper surface and exhibit weaker trend to form soluble complexes with $\mathrm{Cu}^{2+}$ than non-mercapto imidazoles.

As known that the effective inhibitor in neutral solution always do not function in acid solution owing to the different action mechanism. In this regard, it is meaningful to explore the inhibitors that could take effect in two different corrosive media. In our previous research, we investigated that halogenosubstitution on $1 H$-indazole (IA) compound can enhance adsorption intensity and inhibition performance of native IA on copper in a neutral chloride solution. ${ }^{29,30}$ Thus, in this work, we further gave attention to seek experimentally and computationally the inhibition performance of IA and three halogeno-
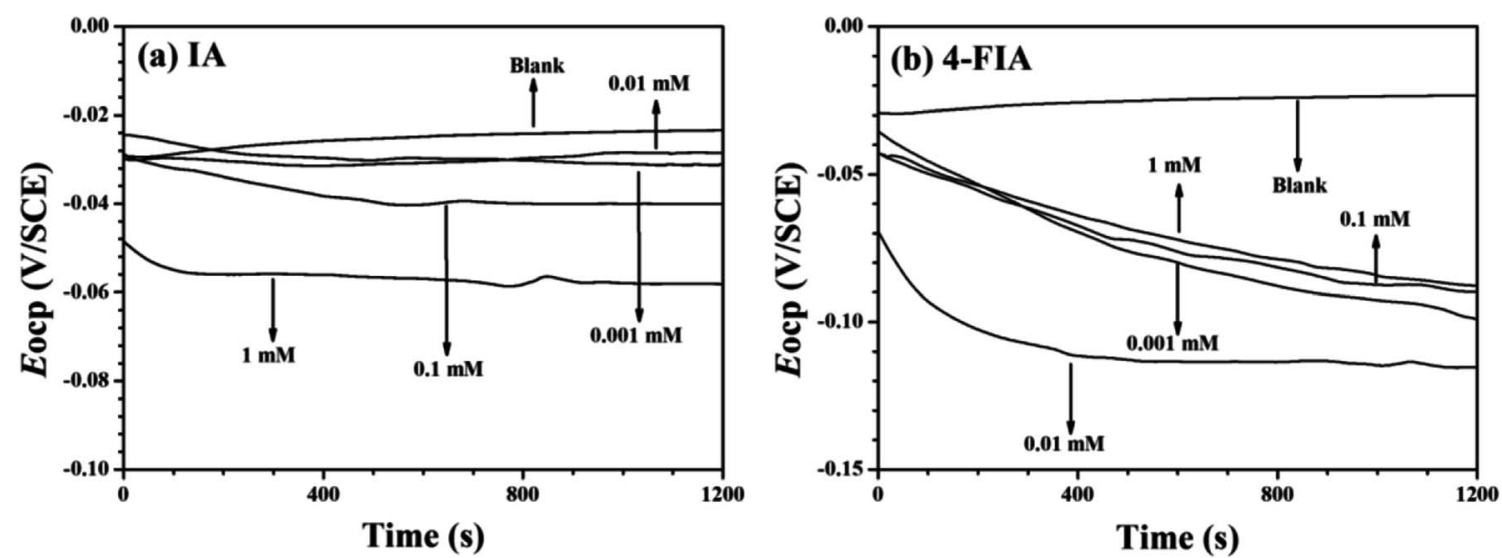

Time (s)
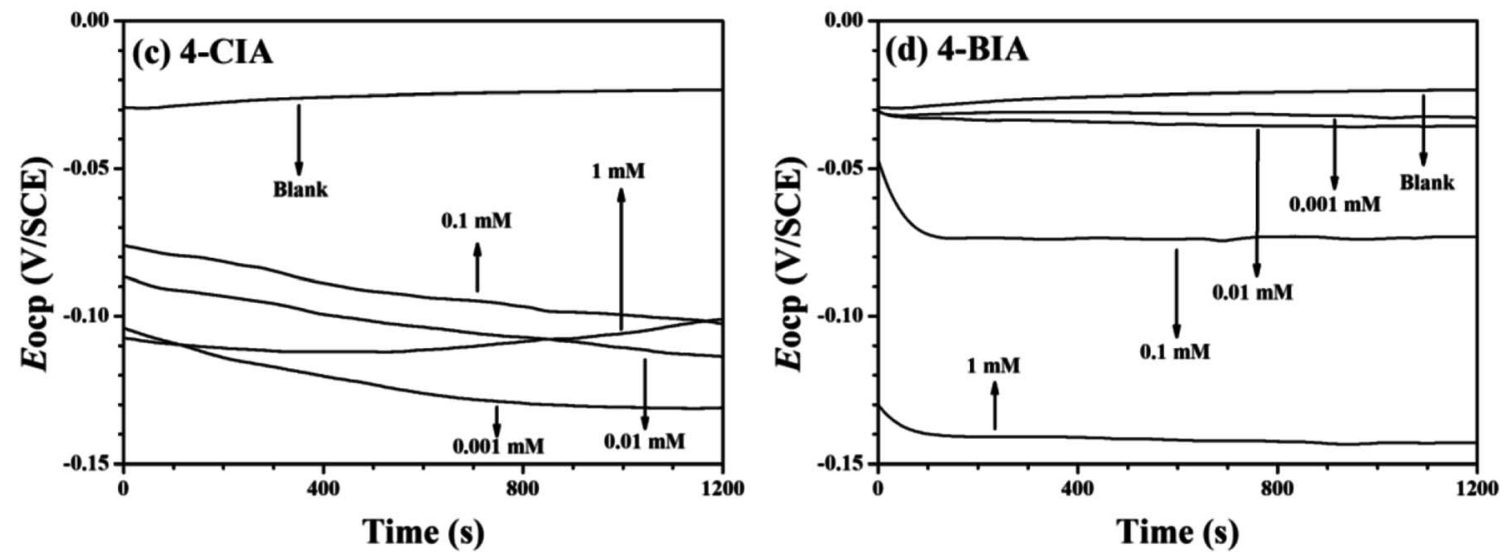

Fig. 2 OCP-time curves for copper in $0.5 \mathrm{~mol} \mathrm{~L}^{-1} \mathrm{H}_{2} \mathrm{SO}_{4}$ solution containing different concentrations of the investigated inhibitors at $298 \mathrm{~K}$, (a) IA, (b) 4-FIA (c) 4-CIA, (d) 4-BIA. 

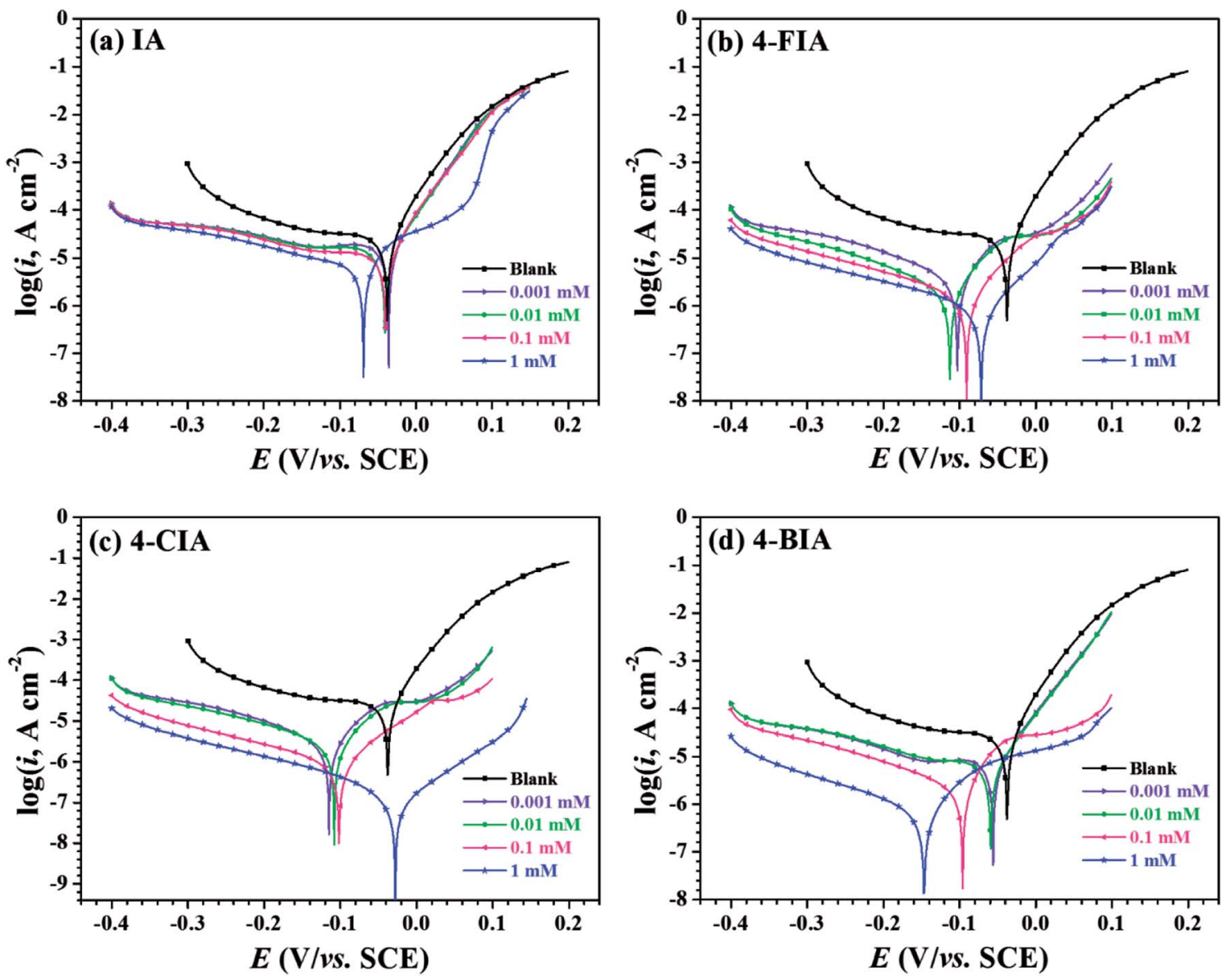

Fig. 3 Potentiodynamic polarization curves recorded for copper in $0.5 \mathrm{M} \mathrm{H}_{2} \mathrm{SO}_{4}$ solution containing different concentrations of the investigated inhibitors at $298 \mathrm{~K}$, (a) IA, (b) 4-FIA (c) 4-CIA, (d) 4-BIA

substituted indazoles (HIAs), 4-fluoro- $1 H$-indazole (4-FIA), 4chloro- $1 H$-indazole (4-CIA), and 4-bromo- $1 H$-indazole (4-BIA), on the corrosion of copper in $0.5 \mathrm{M} \mathrm{H}_{2} \mathrm{SO}_{4}$, which has never been reported before. Potentiodynamic polarization measurement, electrochemical impedance spectroscopy (EIS), field emission scanning electron microscope (FE-SEM), and atomic force microscope (AFM) were adopted to assess the inhibition properties and observe the microstructure of the copper surface. After that, the corresponding inhibition mechanism was captured by theoretical calculation approaches including DFT calculation and molecular dynamics (MD) simulation for protonated state of inhibitors considering solvent effect. Actually, the calculated results shed more light into the molecular reactivity of IA-based inhibitors and the interaction mechanism between these compounds and copper surface in acid solution.

\section{Experimental section}

\subsection{Preparation of materials and solutions}

Testing specimens were cut from a pure copper sheet (99.9\%). For electrochemical tests, the copper coupons $(1.00 \times 1.00 \times$ $1.00 \mathrm{~cm}$ ) were sealed in epoxy, leaving a $1 \mathrm{~cm}^{2}$ area exposed to the aggressive solution. Prior to each experiment, the copper specimens were abraded consecutively with a series of emery papers from 400 to 2000 grit, washed with ultrapure water and acetone, dried at room temperature. Fig. 1 shows the investigated compounds, IA, 4-FIA, 4-CIA, and 4-BIA, which were purchased from Tansool at $99 \%$ purity and used as received. The $0.5 \mathrm{M} \mathrm{H}_{2} \mathrm{SO}_{4}$ solution diluted from analytical grade 98\% $\mathrm{H}_{2} \mathrm{SO}_{4}$ was treated as blank. Then aggressive solution was prepared by $0.5 \mathrm{M}$ sulfuric acid solution without and with various concentrations of studied inhibitors.

\subsection{Electrochemical test}

CHI 760E electrochemical station with a three-electrode system was used to perform the electrochemical measurement. Copper specimen as the working electrode (WE), a big platinum plate as the counter electrode, and a saturated calomel electrode (SCE) as the reference electrode. At first, the WE was immersed in the test solution for $1200 \mathrm{~s}$ at the open circuit potential $\left(E_{\mathrm{OCP}}\right)$ to obtain a steady state condition. Then EIS was conducted on the WE at the $E_{\mathrm{OCP}}$ in the frequency ranging from $100 \mathrm{kHz}$ to $0.01 \mathrm{~Hz}$ with a $10 \mathrm{mV}$ disturbance signal. The EIS data were fitted by Zsimpwin. The inhibition efficiency (IE, \%) was obtained from the polarization resistance $R_{\mathrm{p}}\left(R_{\mathrm{p}}=R_{\mathrm{f}}+R_{\mathrm{ct}}\right)$ as follows,

$$
\mathrm{IE}=\frac{R_{\mathrm{p}}-R_{\mathrm{p}, 0}}{R_{\mathrm{p}}} \times 100
$$


Table 1 Potentiodynamic polarization parameters for copper in $0.5 \mathrm{M} \mathrm{H}_{2} \mathrm{SO}_{4}$ solution without and with various concentrations of four IA-based inhibitors at $298 \mathrm{~K}$

\begin{tabular}{|c|c|c|c|c|c|c|}
\hline$C(\mathrm{mM})$ & $E_{\text {corr }}(\mathrm{mV} / \mathrm{SCE})$ & $i_{\text {corr }}\left(\mu \mathrm{A} \mathrm{cm}^{-2}\right)$ & $\mathrm{SD}^{a}$ & $\beta_{\mathrm{c}}\left(\mathrm{mV} \mathrm{dec}{ }^{-1}\right)$ & $\beta_{\mathrm{a}}\left(\mathrm{mV} \mathrm{dec}{ }^{-1}\right)$ & $\eta(\%)$ \\
\hline Blank & -38 & 28.87 & 3.61 & -483.1 & 42.0 & - \\
\hline 0.001 & -36 & 22.68 & 3.12 & -644.2 & 43.0 & 21.4 \\
\hline 0.01 & -41 & 15.82 & 2.03 & -827.6 & 42.8 & 45.2 \\
\hline 0.1 & -40 & 12.53 & 1.14 & -671.1 & 40.7 & 56.6 \\
\hline \multicolumn{7}{|l|}{ 4-FIA } \\
\hline 0.001 & -103 & 4.81 & 0.56 & -181.0 & 71.1 & 83.3 \\
\hline 0.01 & -113 & 2.83 & 0.33 & -167.3 & 65.9 & 90.2 \\
\hline 0.1 & -91 & 1.88 & 0.27 & -213.4 & 67.3 & 93.5 \\
\hline 1 & -72 & 0.90 & 0.13 & -159.7 & 66.9 & 96.9 \\
\hline 1 & -28 & 0.12 & 0.02 & -123.9 & 81.6 & 99.6 \\
\hline \multicolumn{7}{|l|}{ 4-BIA } \\
\hline 0.001 & -56 & 8.63 & 0.74 & -313.3 & 49.4 & 70.1 \\
\hline 0.01 & -59 & 7.64 & 0.88 & -312.6 & 49.0 & 73.5 \\
\hline 0.1 & -96 & 2.55 & 0.32 & -187.3 & 51.1 & 91.2 \\
\hline 1 & -147 & 0.83 & 0.09 & -183.0 & 74.8 & 97.1 \\
\hline
\end{tabular}

where $R_{\mathrm{p}}$ and $R_{\mathrm{p}, 0}$ are the sum of film resistance and charge transfer resistance of the WE with and without studied organics, respectively. Finally, the potentiodynamic polarization curves were recorded in the potential range of $\pm 250 \mathrm{mV}$ versus the $E_{\text {ocp }}$ at a scan rate of $1 \mathrm{mV} \mathrm{s}^{-1}$. The relevant $\eta$ values were calculated as follows,

$$
\mathrm{IE}=\frac{i_{\text {corr }, 0}-i_{\text {corr }}}{i_{\text {corr }, 0}} \times 100
$$

where $i_{\text {corr, } 0}$ and $i_{\text {corr }}$ denote current densities of uninhibited and inhibited WE, respectively. All tests were conducted at 298 K using a thermostat water bath.

\subsection{Morphology analysis}

The morphology of copper samples before and after exposure to $0.5 \mathrm{M} \mathrm{H}_{2} \mathrm{SO}_{4}$ with and without $1 \mathrm{mM}$ studied inhibitors at $298 \mathrm{~K}$ were observed by AFM (MFP-3D-BIO, Asylum Research, America) and FE-SEM (JEOL-JSM-7800F, JEOL Ltd, Japan).

\subsection{Computational details}

Quantum chemical calculation was conducted using Gaussian 03W software. All of the inhibitor molecules in protonated cationic form shown in Fig. 1 were fully optimized using the B3LYP method for DFT level with a $6-311++G(d, p)$ basis set in aqueous phase. Then, some parameters, including the energy of the lowest unoccupied molecular orbital $\left(E_{\mathrm{LUMO}}\right)$, the energy of the highest occupied molecular orbital ( $\left.E_{\mathrm{HOMO}}\right)$, energy gap $\Delta E$
$\left(\Delta E=E_{\mathrm{LUMO}}-E_{\text {номо }}\right)$, and dipole moment $(\mu)$ were obtained, respectively.

The MD simulation of IA-based compounds on $\mathrm{Cu}$ (111) surface was studied by using the Forcite module from Accelrys Inc. The protonated inhibitor molecules and copper surface was assumed in a simulation box with a COMPASS force field at periodic boundary condition. Both 1 inhibitor and $300 \mathrm{H}_{2} \mathrm{O}$ molecules were allowed to freely interact with the "frozen" $\mathrm{Cu}$ substrate. A NVT canonical ensemble with a time step of 1 fs and simulation time of 500 ps was implemented in this simulation. The interaction energy $\left(E_{\text {interact }}\right)$ between inhibitor molecules and $\mathrm{Cu}$ substrate could be given by: ${ }^{31}$

$$
E_{\text {interact }}=E_{\text {tot }}-\left(E_{\text {subs }}+E_{\text {inh }}\right)
$$

where $E_{\text {tot }}$ is the total energy of the system, $E_{\text {subs }}$ is the energy of copper substrate together with $\mathrm{H}_{2} \mathrm{O}$ molecules, and $E_{\text {inh }}$ is free inhibitor energy. Besides, the binding energy $\left(E_{\text {binding }}\right)$ is the negative value of the interaction energy: ${ }^{31}$

$$
E_{\text {binding }}=-E_{\text {interact }}
$$

\section{Results and discussion}

\subsection{Potentiodynamic polarization curves}

Fig. 2 shows the OCP-time curves for copper in $0.5 \mathrm{M} \mathrm{H}_{2} \mathrm{SO}_{4}$ solution containing various concentrations of the investigated 

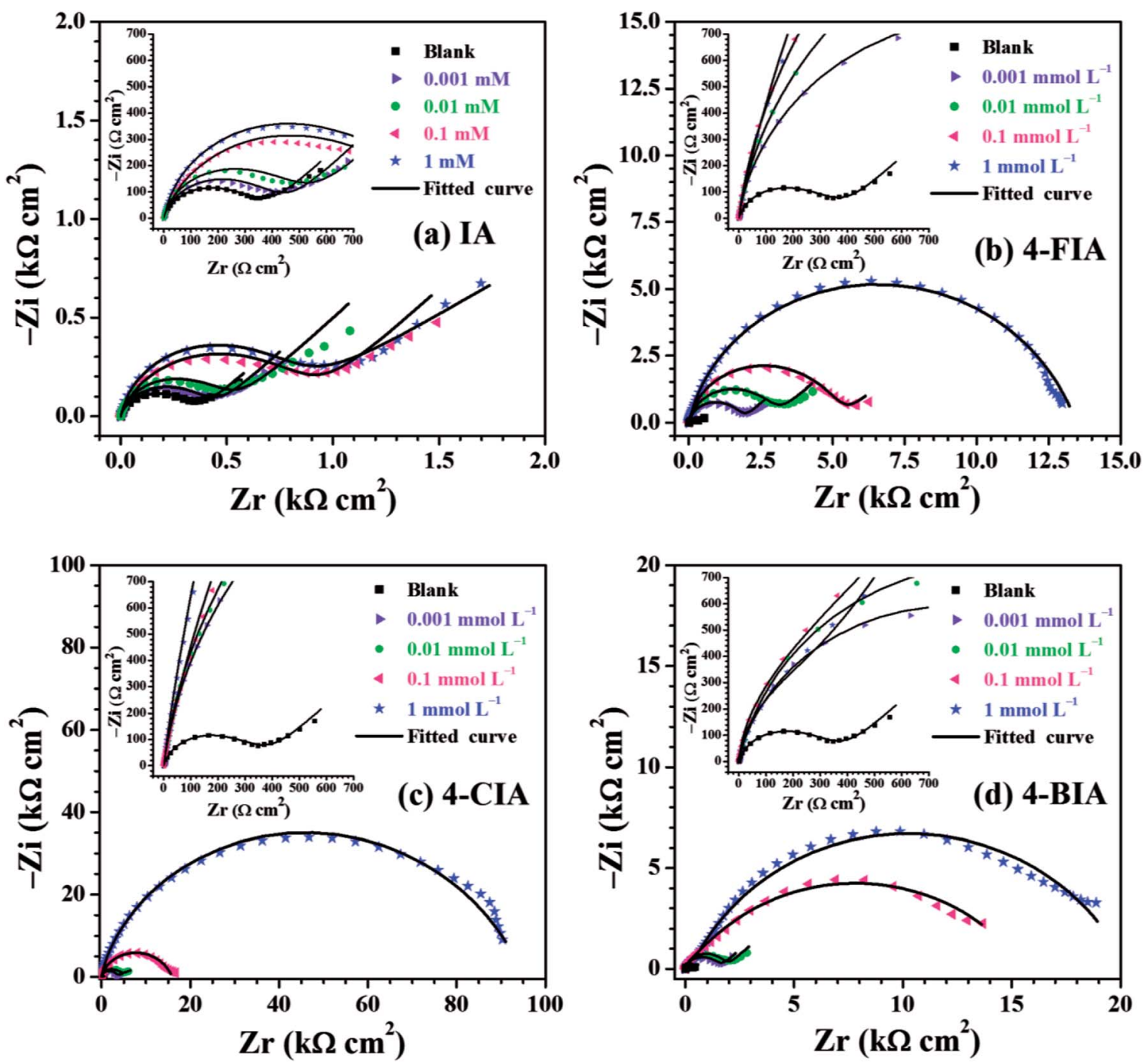

Fig. 4 Nyquist plots for the copper electrode in $0.5 \mathrm{M} \mathrm{H}_{2} \mathrm{SO}_{4}$ solution without and with different concentrations of (a) IA, (b) 4-FIA (c) 4-ClA, (d) $4-\mathrm{BIA}$ at $298 \mathrm{~K}$.

inhibitors at $298 \mathrm{~K}$. As seen that with the addition of these inhibitors, the $E_{\text {ocp }}$ values move to negative values. Fig. 3 illustrates Tafel curves of copper in $0.5 \mathrm{M} \mathrm{H}_{2} \mathrm{SO}_{4}$ with various concentrations of the investigated inhibitors. The relevant electrochemical parameters, including corrosion current density $\left(i_{\text {corr }}\right)$, corrosion potential $\left(E_{\text {corr }}\right)$, anodic Tafel slope $\left(\beta_{\mathrm{a}}\right)$, cathodic Tafel slope $\left(\beta_{\mathrm{c}}\right)$, and inhibition efficiency (IE) are obtained and shown in Table 1 . These parameters and are shown in Table 1, respectively. As seen in Fig. 3 that all of the curves move to lower current densities in the presence of four inhibitors, compared to the uninhibited solution. This phenomenon is more pronounced at higher inhibitor concentrations, indicating that the addition of all studied compounds in whole concentration range impedes the corrosion rate of copper.

Specifically, all cathodic branches of the polarization curves in the presence of inhibitors are parallel with that of blank, which indicates that the mechanism of cathodic reaction is not changed by these organic compounds and the corrosion processes are retarded by reducing the reaction rates. ${ }^{32}$ For another, the anodic branches of obtained polarization curves appear more complicated. At low inhibitor concentrations of IA and 4-BIA where the anodic curves vary slightly, the geometric blocking effect dominates on the inhibition performance, while in the high concentration range of IA and 4-BIA, the active blocking effect plays a main role by the formation of anchored adsorption films. ${ }^{33}$ Especially, 4-FIA and 4-CIA present active blocking mechanism in the whole concentration range.

As seen in Table 1, except negative movement of the $E_{\text {corr }}$ values for $1 \mathrm{mM} 4$-BIA, all of the displacements are less than $85 \mathrm{mV}$ compared with the blank. It can be deduced that 4-BIA acts as a modest cathodic inhibitor, while other inhibitors were mixed-type inhibitors. ${ }^{34}$ The cathodic Tafel slope $\left(\beta_{c}\right)$ values have a little difference, whereas some $\beta_{\mathrm{a}}$ values change a lot with the addition of the inhibitors, which indicate the complicated anodic electrode process mentioned above. Additionally, the $i_{\text {corr }}$ values shown in Table 1 decrease significantly with increasing inhibitor concentration, leading to the simultaneous increase of inhibition efficiency up to $66.4 \%$ (IA), 96.9\% (4-FIA), 

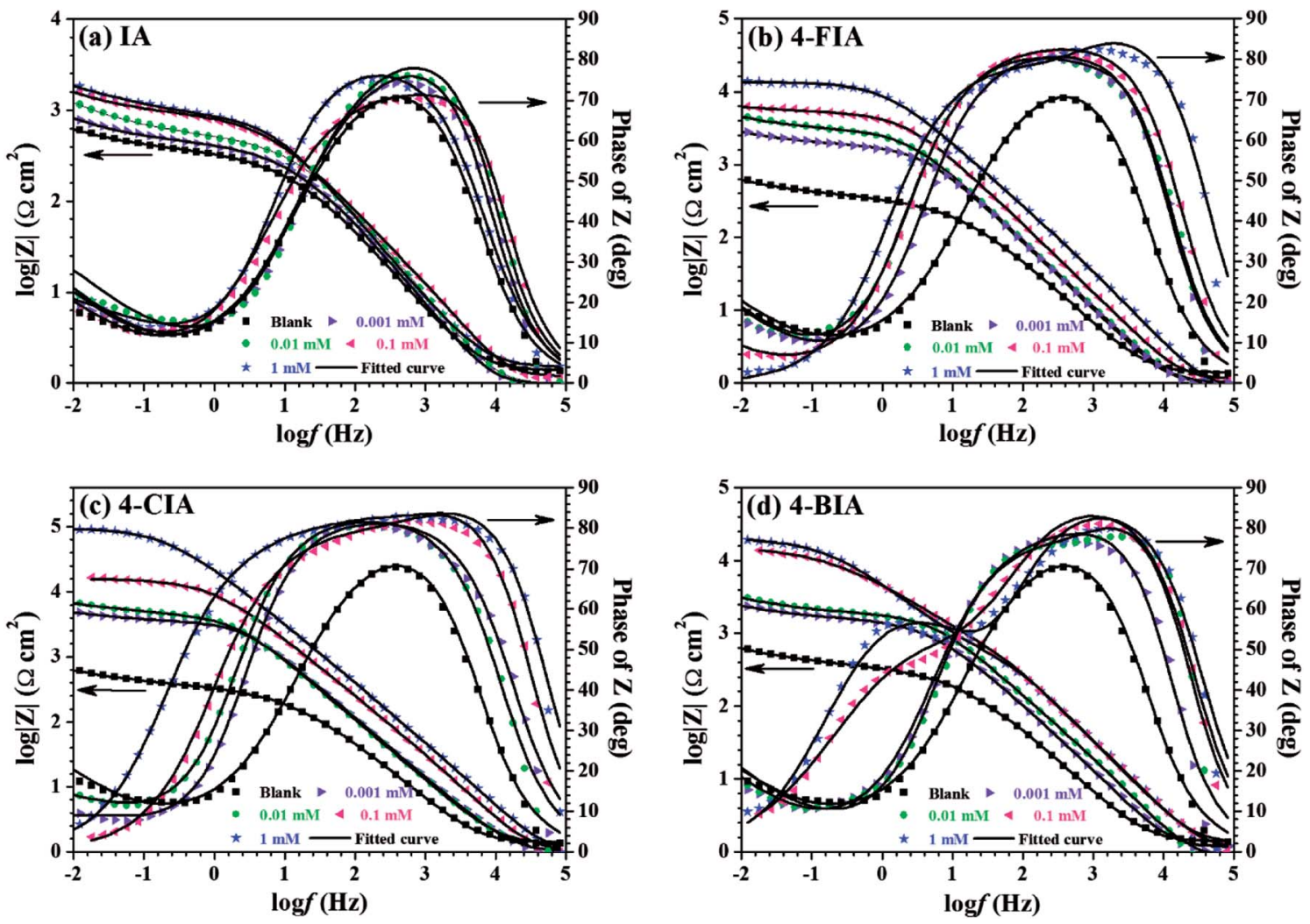

Fig. 5 Bode graphs of the measured impedance shown in Fig. 3, (a) IA, (b) 4-FIA (c) 4-CIA, (d) 4-BIA.

(a)

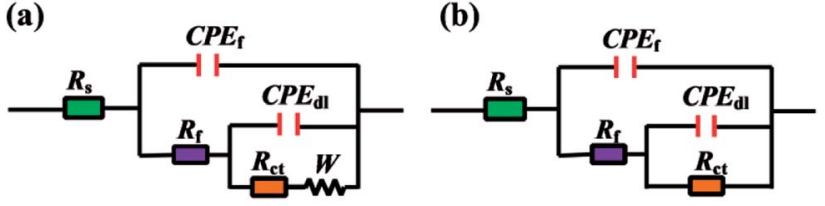

Fig. 6 Equivalent circuits used to fit the EIS experimental data.

99.6\% (4-CIA), and 97.1\% (4-BIA), respectively. Thus, it is worth mentioning that the efficiencies of all HIAs are far more than IA inhibitor and 4-CIA are greatest. Considering the multiplicity of adsorption centres in studied HIAs than IA molecule itself, it is reasonable to deduce that halogeno-substitution strengthen the adherence of the inhibitor film.

\subsection{EIS measurements}

The relevant Nyquist diagram for copper in $0.5 \mathrm{M} \mathrm{H}_{2} \mathrm{SO}_{4}$ medium with and without various concentrations of studied IAbased inhibitors are shown in Fig. 4. For the blank solution, Fig. 4 shows a depressed capacitive semicircle in high frequency (HF) followed by a straight line in low frequency (LF) range. Generally, the semicircle is attributed to a charge-transfer process of the copper dissolution, ${ }^{35}$ while the LF straight line is Warburg impedance due to either the diffusion of dissolved oxygen from bulk solution to the copper surface or the transport of corrosive particles. ${ }^{36}$ The diameter of the capacitive arc increases slightly by introducing IA, in comparison with that obtained in the blank, representing an inferior inhibition performance of IA. Nevertheless, the addition of three HIAs remarkably increase the diameter of the curves, which is associated with high inhibitive ability. It can be inferred that more stable protective films of HIAs are formed on copper surface than that of IA. The capacitive loops increase continuously with the increasing inhibitor concentration, indicating that the inhibitor films are gradually compact. Besides, Warburg impedance disappears in high concentrations of three HIAs, which suggests that these compounds strongly retard the diffusion process. ${ }^{5}$ On the other hand, there is still diffusive impedance for IA and low concentration of HIAs owing to the relatively loose inhibitor layer in this case. ${ }^{37}$ The results reveal the formation of the inhibitor-adsorption films on surface of copper and their contribution to the increase of anti-corrosive quality.

From Bode plots (Fig. 5), with the addition and increasing concentration of IA, the impedance modulus increases compared to that obtained in the blank solution, while this phenomenon is clearer for 4-FIA, 4-CIA, and 4-CIA. Besides, the maximum phase angle increases with increasing inhibitor concentration and HIAs harbour higher value of phase angle than IA. These results manifests higher inhibitive ability of HIAs than single IA for copper corrosion.

The equivalent circuits shown in Fig. 6 were used to fit the impedance data in present study. $\mathrm{CPE}_{\mathrm{f}}$ and $\mathrm{CPE}_{\mathrm{dl}}$ represent constant phase angle elements, which are related to double layer capacitance $\left(C_{\mathrm{dl}}\right)$, film capacitance $\left(C_{\mathrm{f}}\right)$, respectively. The use of CPE instead a pure capacitor introduces non-ideal 
Table 2 Impedance parameters for copper in $0.5 \mathrm{M} \mathrm{H}_{2} \mathrm{SO}_{4}$ solution in the absence and presence of different concentrations of four IA-based compounds at $298 \mathrm{~K}$

\begin{tabular}{|c|c|c|c|c|c|c|c|c|c|c|}
\hline$C(\mathrm{mM})$ & $R_{\mathrm{f}}\left(\Omega \mathrm{cm}^{2}\right)$ & $R_{\mathrm{ct}}\left(\mathrm{k} \Omega \mathrm{cm}^{2}\right)$ & $R_{\mathrm{p}}\left(\mathrm{k} \Omega \mathrm{cm}^{2}\right)$ & $C_{\mathrm{f}}\left(\mu \mathrm{F} \mathrm{cm}^{-2}\right)$ & $n_{1}$ & $C_{\mathrm{dl}}\left(\mu \mathrm{F} \mathrm{cm}^{-2}\right)$ & $n_{2}$ & $\begin{array}{l}W \\
\left(\times 10^{-2} \Omega \mathrm{cm}^{2} \mathrm{~s}^{1 / 2}\right)\end{array}$ & $\eta(\%)$ & $\begin{array}{l}\chi^{2} \\
\left(\times 10^{-2}\right)\end{array}$ \\
\hline Blank & 7.8 & 0.40 & $0.41 \pm 0.02$ & 33.2 & 0.93 & 43.0 & 0.43 & 1.31 & - & 0.62 \\
\hline \multicolumn{11}{|l|}{ IA } \\
\hline 0.001 & 13.6 & 0.48 & $0.49 \pm 0.02$ & 19.4 & 1 & 31.4 & 0.57 & 0.87 & 16.3 & 0.54 \\
\hline 0.01 & 29.3 & 0.51 & $0.54 \pm 0.03$ & 15.9 & 1 & 20.5 & 0.57 & 0.49 & 24.1 & 0.83 \\
\hline \multicolumn{11}{|l|}{ 4-FIA } \\
\hline 0.001 & 40.3 & 1.79 & $1.83 \pm 0.05$ & 14.2 & 1 & 13.5 & 0.72 & 0.32 & 77.6 & 0.66 \\
\hline 0.01 & 39.5 & 2.89 & $2.93 \pm 0.07$ & 12.9 & 1 & 13.6 & 0.76 & 0.20 & 86.0 & 0.53 \\
\hline 0.1 & 41.8 & 5.25 & $5.29 \pm 0.06$ & 8.2 & 1 & 8.7 & 0.71 & 0.31 & 92.2 & 0.49 \\
\hline 1 & 247.1 & 13.2 & $13.5 \pm 0.10$ & 4.5 & 1 & 6.9 & 0.73 & - & 97.0 & 1.02 \\
\hline 0.1 & 210.7 & 15.7 & $15.9 \pm 0.09$ & 4.9 & 1 & 7.8 & 0.70 & - & 97.4 & 0.90 \\
\hline 1 & 270.3 & 95.2 & $95.5 \pm 0.18$ & 3.1 & 1 & 6.6 & 0.72 & - & 99.6 & 0.45 \\
\hline \multicolumn{11}{|l|}{ 4-BIA } \\
\hline 0.001 & 41.2 & 1.55 & $1.59 \pm 0.03$ & 11.2 & 1 & 15.9 & 0.66 & 0.37 & 74.2 & 0.77 \\
\hline 0.01 & 85.1 & 1.78 & $1.87 \pm 0.03$ & 7.7 & 1 & 10.3 & 0.74 & 0.26 & 78.1 & 0.82 \\
\hline 0.1 & 142.1 & 14.9 & $15.0 \pm 0.07$ & 6.0 & 0.97 & 9.2 & 0.71 & - & 97.3 & 1.10 \\
\hline 1 & 174.0 & 19.7 & $19.9 \pm 0.10$ & 6.1 & 0.97 & 8.7 & 0.73 & - & 97.9 & 0.98 \\
\hline
\end{tabular}

dielectric behaviour for the inhomogeneous electrode surface. $^{38,39}$ The obtained impedance parameters including charge transfer resistance $\left(R_{\mathrm{ct}}\right)$, film resistance $\left(R_{\mathrm{f}}\right)$, Warburg impedance $(W), C_{\mathrm{dl}}, C_{\mathrm{f}}$, and so on are summarized in Table 2. The $C_{\mathrm{dl}}$ and $C_{\mathrm{f}}$ values are calculated as follows, ${ }^{40}$

$$
C=Y_{0}\left(w_{\max }\right)^{n-1}
$$

where $Y_{0}$ is the magnitude of the CPE, $w_{\max }=2 \pi f_{\max }$ and $f_{\max }$ is the frequency at the maximum value of the imaginary component of the impedance spectrum, and $n$ is the deviation parameter in regard to the phase shift.

As seen in Table 2 that the addition of the four inhibitors increases both the $R_{\mathrm{ct}}$ and $R_{\mathrm{f}}$ values and increasing concentration enhance this trend, indicating that the formation of IAsadsorption films mitigate the process of charge transfer.
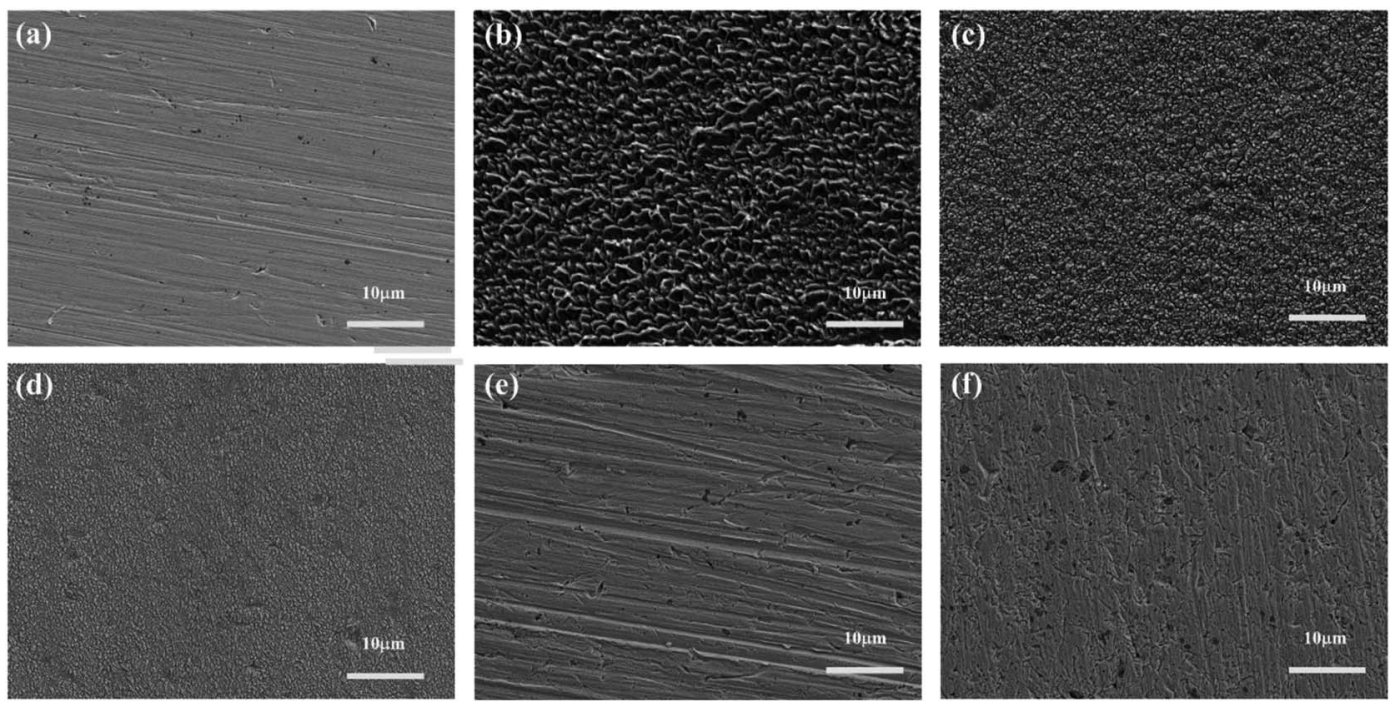

Fig. 7 FE-SEM images of (a) freshly polished copper specimen and the specimens immersed in $0.5 \mathrm{M} \mathrm{H}_{2} \mathrm{SO}_{4}$ solution (b) without and with 1 mM (c) IA, (d) 4-FIA, (e) 4-CIA, (f) 4-BIA for $32 \mathrm{~h}$ at $298 \mathrm{~K}$. 
(a)

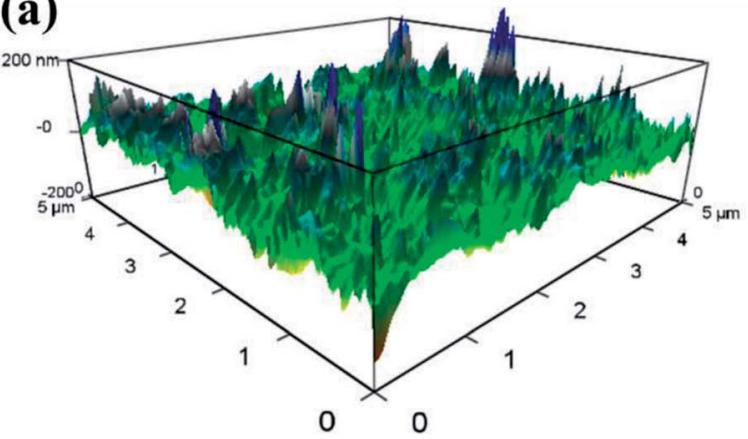

(b)

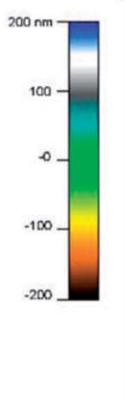

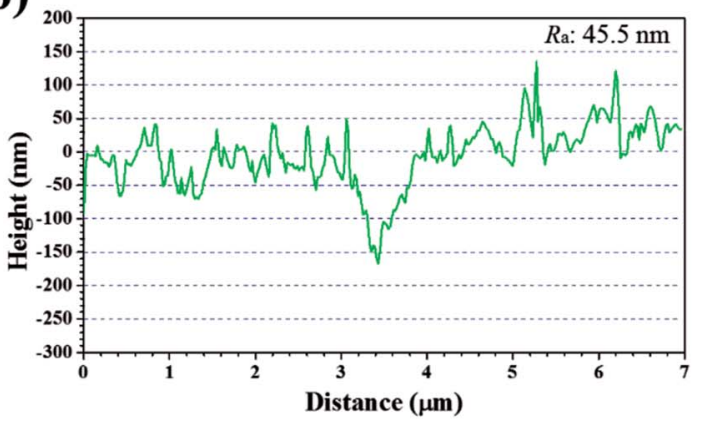

Fig. 8 (a) 3D AFM graph and (b) height profile of copper specimen after immersion in $0.5 \mathrm{M} \mathrm{H}_{2} \mathrm{SO}_{4}$ solution without inhibitors for $8 \mathrm{~h}$ at $298 \mathrm{~K}$.

(a)

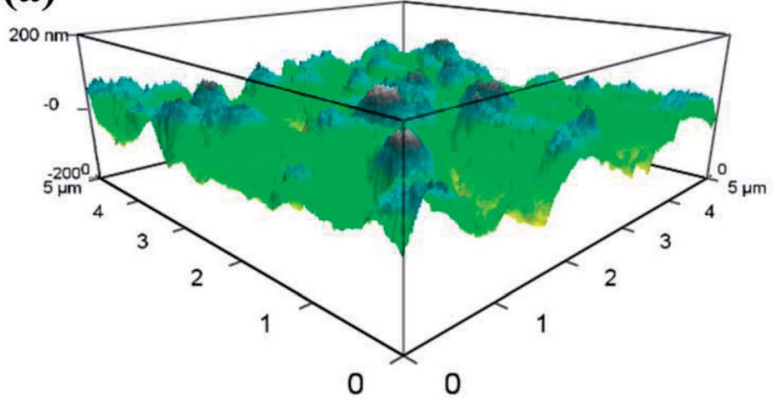

(b)

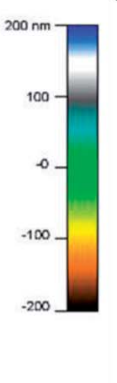

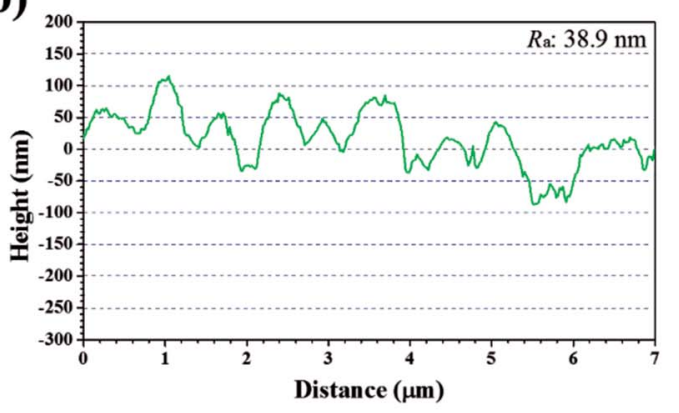

Fig. 9 (a) 3D AFM graph and (b) height profile of copper specimen after immersion in $0.5 \mathrm{M} \mathrm{H}_{2} \mathrm{SO}_{4}$ solution in the presence of $1 \mathrm{mM} \mathrm{IA} \mathrm{for} 8 \mathrm{~h}$ at $298 \mathrm{~K}$.

Impressively, this phenomenon is remarkable for three HIAs than original IA, implying the more stable films of HIAs. Furthermore, the values of $C_{\mathrm{f}}$ and $C_{\mathrm{dl}}$ decrease with the addition of four IA-based organics, which is owing to the replacement of water molecules by investigated inhibitor molecules. ${ }^{41}$ Accordingly, the IE values of these IA-based compounds increase with increasing inhibitor concentration and reach $57.3 \%$ for IA, $97.0 \%$ for 4 -FIA, $99.6 \%$ for 4 -CIA, and $97.9 \%$ for 4 -BIA at $1 \mathrm{mM}$, respectively. The order of inhibition ability agrees well with the results gained from the polarization curves and reveals that HIAs provide more effective protection against copper corrosion in $0.5 \mathrm{M} \mathrm{H}_{2} \mathrm{SO}_{4}$ medium than native IA. Moreover, the maximum IE value of 4-CIA are higher than that of the corrosion inhibitors in same condition reported before, such as $81 \%$ of indole-3-carboxylic acid, ${ }^{18}$ 94.0\% of rhodanine- $\mathrm{N}$-acetic acid, ${ }^{19}$ $98.3 \%$ of cyproconazole,$^{36}$ and $93.7 \%$ of arginine. ${ }^{23}$

\subsection{Surface observation}

The SEM micrographs of copper surface before and after $32 \mathrm{~h}$ immersion in $0.5 \mathrm{M} \mathrm{H}_{2} \mathrm{SO}_{4}$ in the absence and presence of $1 \mathrm{mM}$ IA-based inhibitors at $298 \mathrm{~K}$ are shown in Fig. 7. The freshly polished copper surface (Fig. 7a) is smooth, whereas the uninhibited copper surface (Fig. 7b) shows obvious deterioration due to the aggressive attack by corrosive particles. A slightly smoother surface is obtained in Fig. 7c in the presence of IA, (a)

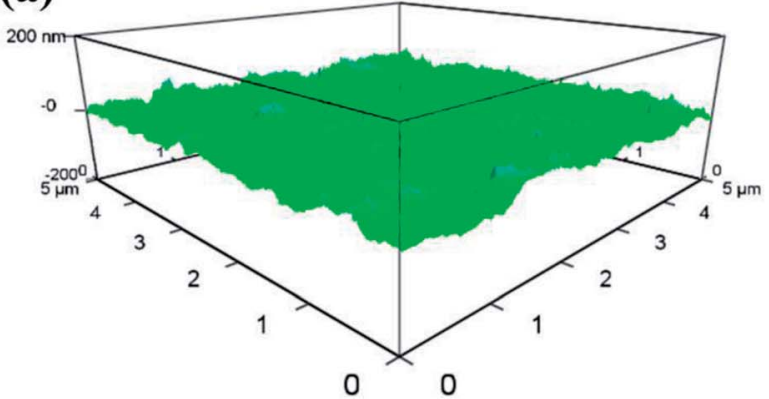

(b)

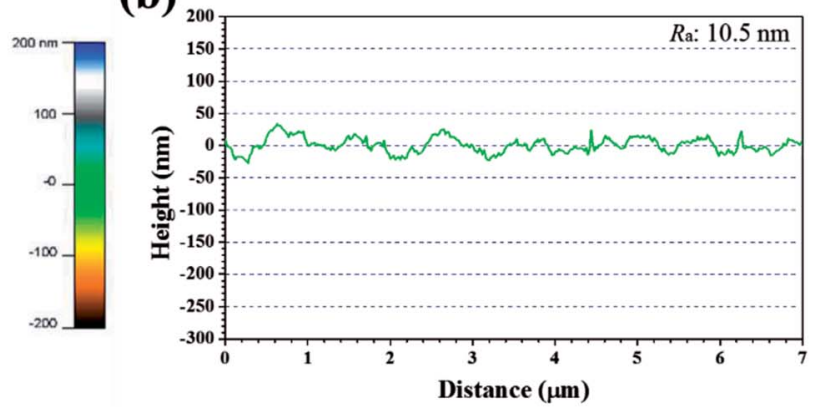

Fig. 10 (a) 3D AFM graph and (b) height profile of copper specimen after immersion in $0.5 \mathrm{M} \mathrm{H}_{2} \mathrm{SO}_{4}$ solution in the presence of $1 \mathrm{mM} 4$ - $\mathrm{ClA}$ for $8 \mathrm{~h}$ at $298 \mathrm{~K}$. 


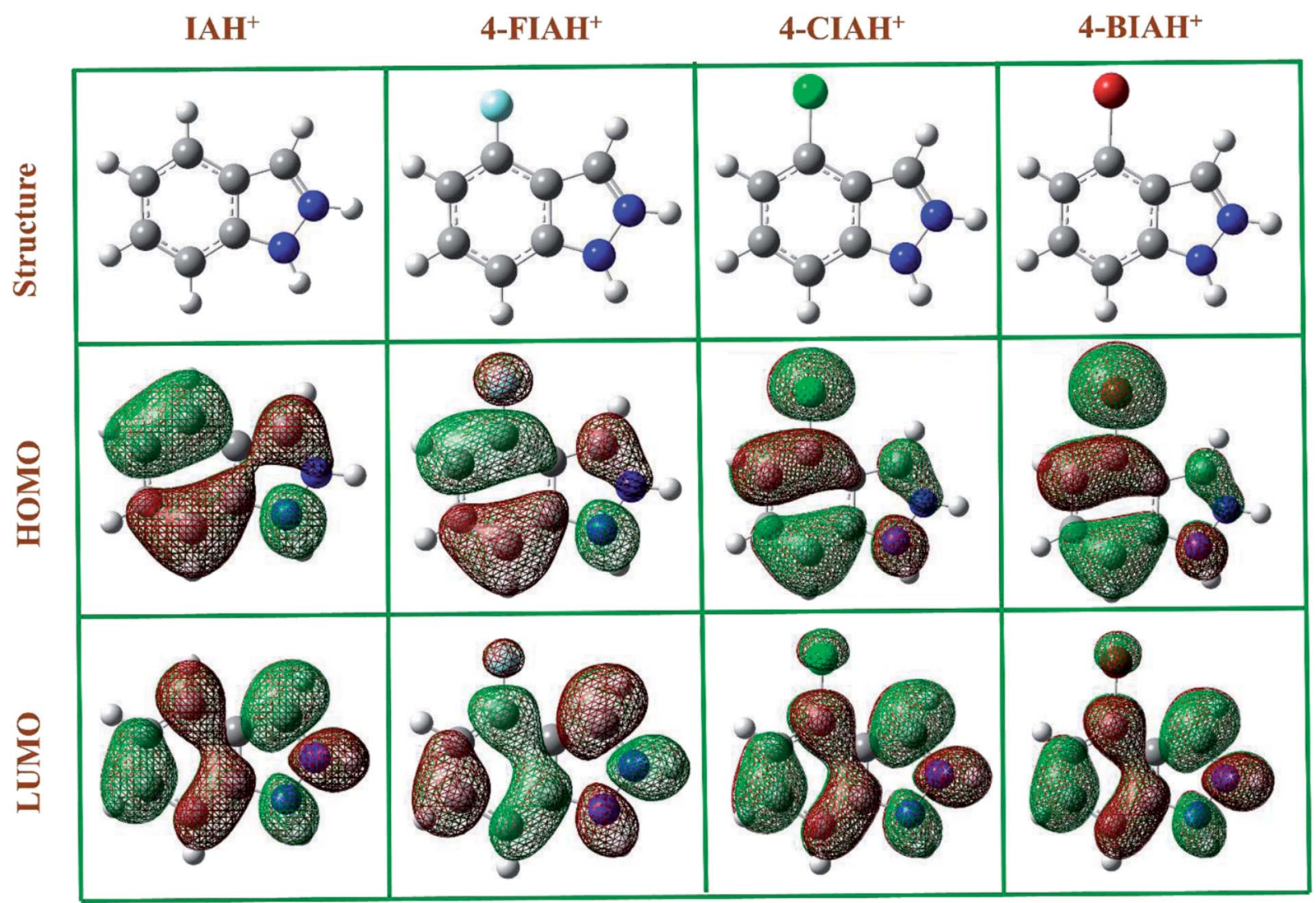

Fig. 11 The optimized structures, frontier orbital density distributions of four protonated IA-based compounds in liquid phase.

whereas Fig. $7(\mathrm{~d}-\mathrm{f})$ with the addition of HIAs exhibit quite smooth surfaces as expected. Particularly, the surface protected by 4-CIA is the flattest surface and nearly same as the surface in Fig. 7a. These observations indicate that inhibitor films are formed on surface of copper, therefore inhibited surface has lower permeability for aggressive particles than uninhibited copper surface. Consequently, the copper surface was effectively protected by HIAs, especially 4-CIA, which supports the electrochemical results convincingly.

Recent years, AFM has been widely applied in corrosion field to observe surface appearance at the nano- to microscale level. $^{34,42-45}$ The 3D AFM images and corresponding height profiles of unprotected and protected copper surfaces with $1 \mathrm{mM}$ IA and 4-CIA respectively in $0.5 \mathrm{M} \mathrm{H}_{2} \mathrm{SO}_{4}$ solution at $298 \mathrm{~K}$ for $8 \mathrm{~h}$ are shown in Fig. 8-10. For copper surfaces protected by 4-FIA and 4-BIA respectively, the corresponding AFM images are given in Fig. S1 and S2. $\dagger$ It is noticeable in Fig. 8a that corroded copper sample shows considerably rough structure with deep and large pits owing to the aggressive attack. This appearance can be further evidenced by the height profile in Fig. 8b, which exhibits large peaks and valleys about $200 \mathrm{~nm}$ height. Moreover, the average roughness $\left(R_{\mathrm{a}}\right)$ of the corroded copper surface shown in Fig. 8b is $45.5 \mathrm{~nm}$. In the presence of IA (Fig. 9), the surface becomes slightly flat and relevant $R_{\mathrm{a}}$ decreases to $38.9 \mathrm{~nm}$, while the graph of 4-CIA (Fig. 10) provides a more uniform surface and has a slight fluctuation within $30 \mathrm{~nm}$. As Fig. S1 and S2 $\uparrow$ show, with the addition of 4-FIA and 4-BIA, the
AFM graph are obtained exactly like that of 4-CIA. Besides, the corresponding values of $R_{\mathrm{a}}$ after introducing these HIAs are reduced to $14.5 \mathrm{~nm}$ for 4-FIA, $10.5 \mathrm{~nm}$ for 4 -CIA, and $11.8 \mathrm{~nm}$ for 4-BIA. These results suggest that the inhibitive ability of the studied compounds obeys the rank of 4-CIA $>4$-BIA $>4$-FIA $>$ IA, which is consistent with the order of electrochemical inhibition efficiencies above.

FE-SEM and AFM observation combined with the electrochemical results demonstrate that denser protective barriers could be formed by HIAs than IA, so that copper is availably protected. Particularly, 4-CIA adsorption-film possessing the highest stability acts as the most powerful shield for corrosion of copper.

\subsection{Quantum chemical study}

The optimized structures, the lowest unoccupied molecular orbital (LUMO), and the highest occupied molecular orbital (HOMO) density distribution of studied IA-based organics for protonated species are presented in Fig. 11. Indeed, some of the calculated quantum chemical properties, including $E_{\text {Номо, }}$ $E_{\text {LUMO }}$, as well as $\Delta E$ are shown graphically in Fig. 12. Fig. 11 shows that electronic density distributions of the four IA-based compounds are concentrated evenly over the whole molecule, which could be related to the high electron densities of the $-\mathrm{N}-$ $\mathrm{N}=\mathrm{C}-$ group, benzene ring, and halogen atoms. This kind of system infers that a flat-adsorption configuration may obtain to 


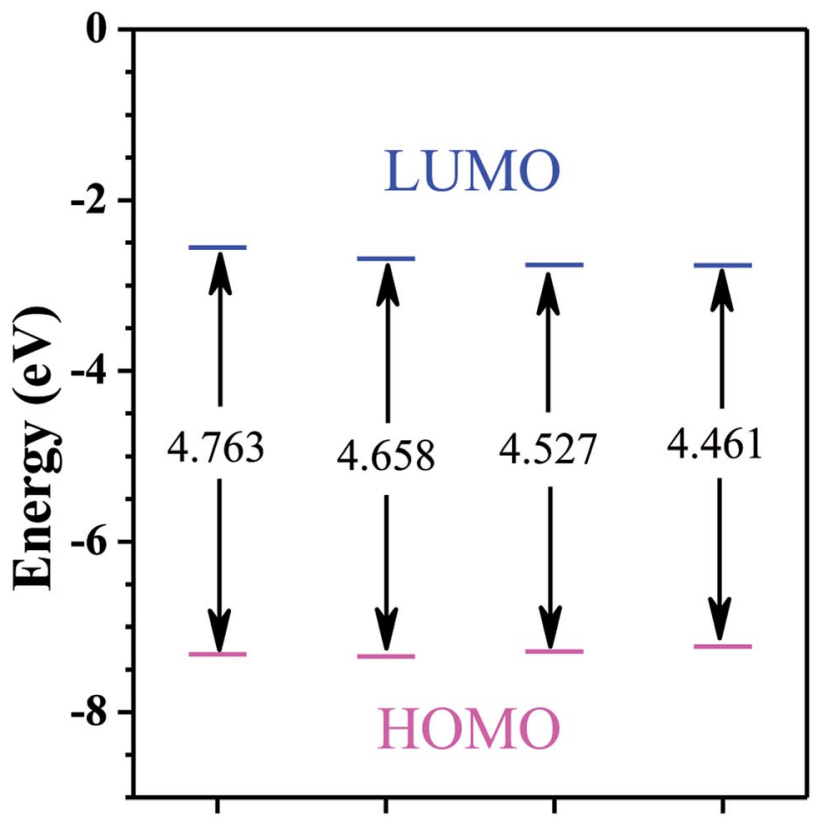

IAH $^{+}{ }^{4-F I A H}{ }^{+}$4-CIAH ${ }^{+}$4-BIAH ${ }^{+}$

Fig. 12 The diagram of calculated parameters $E_{\mathrm{HOMO}}, E_{\mathrm{LUMO}}$, and $\Delta E$ for protonated species of studied inhibitor molecules in liquid phase. gain the largest protective area for copper. It is recognized that $E_{\text {LUMO }}$ represents the electron-receiving ability of the organic molecule, and a low value of $E_{\mathrm{LuMO}}$ is an index of the strong electron-receiving tendency. ${ }^{46} E_{\text {Hомо, }}$ on the other hand, signifies an electron-donating ability of a molecule, and a high value of $E_{\text {номо }}$ illustrates that the inhibitor tends to donate electrons to a suitable acceptor with an empty molecular orbital and low $E_{\text {LUMO }}{ }^{46}$ The lower $E_{\text {LUMO }}$ and higher $E_{\text {HOMO values of }}$ HIA molecules shown in Fig. 12, implying stronger electron acceptance and donation abilities of these molecules, thus owning processing higher inhibition efficiency than IA. Generally, a lower $\Delta E$ means greater adsorption on metal surface. ${ }^{47}$ The calculated $\Delta E$ values of studied inhibitors follow the order: 4-BIA $<4$-CIA $<4$-FIA < IA, which disagree with the electrochemical results to some extent. This phenomenon can be interpreted that the largest radius of $\mathrm{Br}$ influences its adsorption strength and thus reduces its inhibition performance.

There is lack of agreement on the correlation between $\mu$ and inhibitive ability. ${ }^{48}$ According to some authors, a low $\mu$ value will favour accumulation of the inhibitor on metal surface, resulting in an increase of inhibition effectiveness. ${ }^{\mathbf{4 1}}$ However, others proposed that a high value of $\mu$ can enhance the adsorption strength on the surface of metal due to high electrical polarity, thus increasing the inhibition efficiency. ${ }^{\mathbf{4 4}}$ The order of $\mu$ values
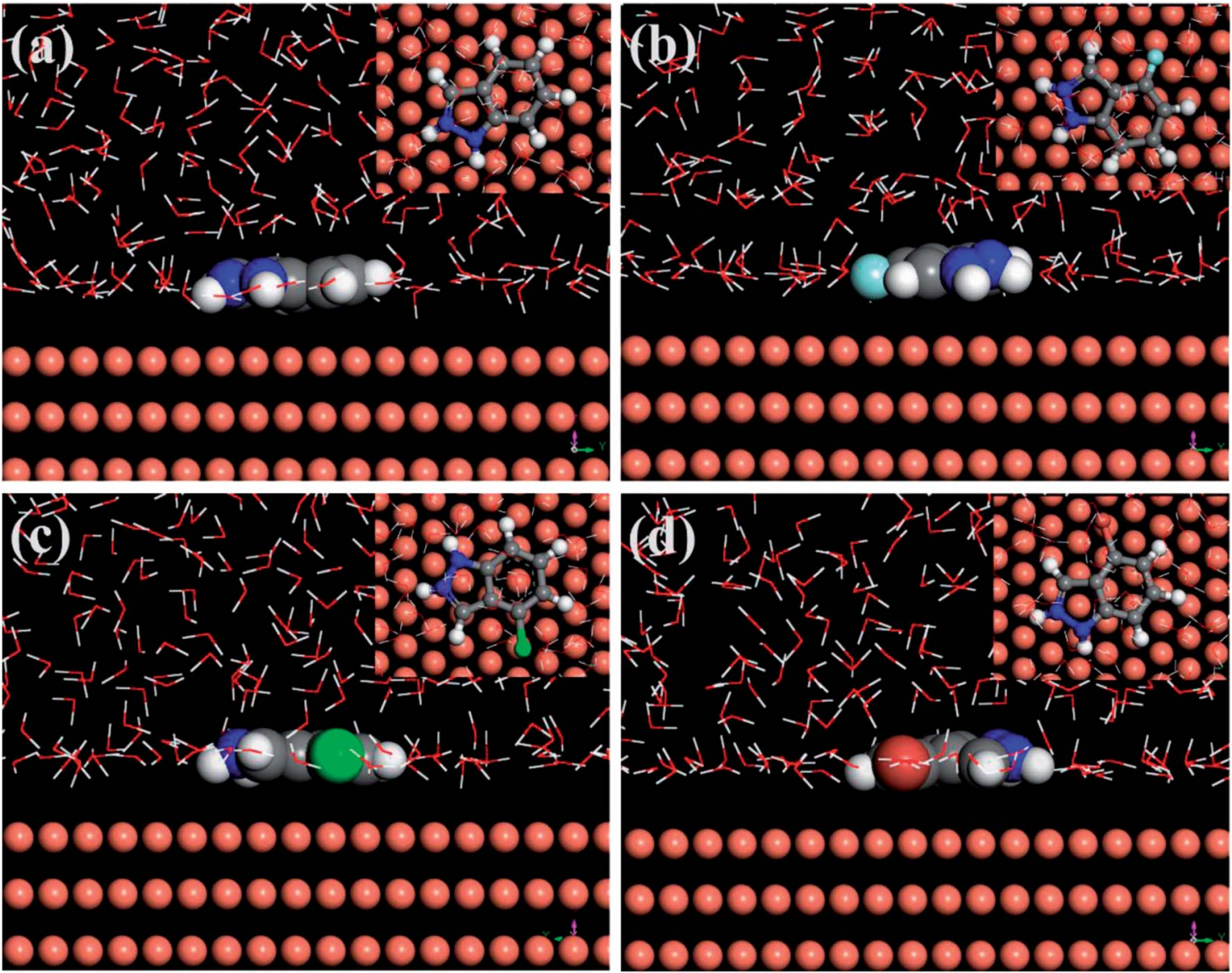

Fig. 13 Equilibrium configurations for the adsorption of protonated inhibitor molecules on the $\mathrm{Cu}$ (111) surface (inset: on-top views), (a) IAH ${ }^{+}$, (b) 4-FIAH ${ }^{+}$, (c) 4- $\mathrm{CIAH}^{+}$, (d) 4-BIAH ${ }^{+}$ 
IA (7.227 D) < 4-FIA (9.265 D) < 4-CIA (10.399 D) < 4-BIA (12.491 D) seemly agrees with the latter viewpoint, which is not well accordance with their inhibition efficiencies above. Therefore, the DFT approach failed to explain the corrosion inhibition rating well due to no consideration of the interaction between inhibitor molecules and copper substrate, whereas it still provided useful molecular properties contributing to understand the inhibition mechanism.

\subsection{Molecular dynamics simulation}

The MD simulation was designed to predict the most stable adsorption configuration and the binding strength of the four protonated IA-based compounds on copper substrate in order to further explain the corresponding mechanism of corrosion inhibition. The side and top views of the equilibrium adsorption configurations of the four investigated inhibitor molecules on $\mathrm{Cu}$ (111) surface are depicted in Fig. 13. It can be seen that all of the four inhibitors adsorb tightly onto the copper surface with a parallel orientation owing to the distribution of FMO densities on whole molecules. This implies that the coordination bond could form through HOMO of the inhibitors to unfilled $\mathrm{sp}^{3}$ non-equivalent hybrid orbital of $\mathrm{Cu}$ (electrondonation) and the transfer of electrons from the $3 \mathrm{~d}$-orbital of $\mathrm{Cu}$ to the vacant orbital of inhibitor molecules (antibonding). ${ }^{\mathbf{4 9 5 0}}$ Importantly, the parallel adsorption configuration can control the aggressive attack availably by minimizing the contact area between $\mathrm{Cu}$ and corrosive particles.

Generally, organic molecules adsorb on a metal surface blocking the cathodic and anodic sites. Hence, more negative the interaction energy or more positive the binding energy between the adsorbed molecule and the metal surface is, higher is the inhibition efficiency. ${ }^{23,51}$ The calculated $E_{\text {binding values are }}$ $230.21 \mathrm{~kJ} \mathrm{~mol}^{-1}$ for $\mathrm{IAH}^{+}, 239.84 \mathrm{~kJ} \mathrm{~mol}^{-1}$ for $4-\mathrm{FIAH}^{+}$, $255.29 \mathrm{~kJ} \mathrm{~mol}^{-1}$ for $4-\mathrm{CIAH}^{+}$, and $253.32 \mathrm{~kJ} \mathrm{~mol}^{-1}$ for $4-\mathrm{BIAH}^{+}$. It is apparent that there is well positive correlation between the experimental inhibition efficiencies of the studied IA-based inhibitors and the relevant binding energies. In particular, protonation of the organic molecules gives rise to slightly larger $E_{\text {binding }}$ values than those obtained for relevant neutral inhibitor molecules, but the two forms follow the same trend for the parameter. ${ }^{30}$ Thus, the favourable verification and explanation for the effectiveness of investigated inhibitors for copper corrosion in two different media, implying that such method can be applied as a computational protocol to convincingly forecast the inhibitive performance rating of organic inhibitors.

\section{Conclusions}

In the case of four IA-based compounds namely IA, 4-FIA, 4-BIA, and 4-BIA as effective inhibitors for copper corrosion in $0.5 \mathrm{M}$ $\mathrm{H}_{2} \mathrm{SO}_{4}$ medium, systematic and comprehensive means were employed to investigate the inhibitive ability and the corresponding mechanism. The following points can be emphasized:

(1) The polarization curve results reveal that 4-BIA acts as modest cathodic inhibitor, while other inhibitors could be considered as mixed-type inhibitors that suppress both cathodic and anodic reactions.

(2) Electrochemical tests indicate that, the inhibition efficiencies increase with incremental concentration and all HIAs possess superior inhibitive ability than native IA. The specific rating of inhibition performance follows the order: IA $<4$-FIA $<$ 4 -BIA < 4-CIA. All IE values of HIAs obtained upon $96 \%$ in $1 \mathrm{mM}$, especially, 4-CIA reach $99.6 \%$.

(3) The rating of inhibition efficiencies obtained electrochemically was further confirmed by FE-SEM and AFM observation.

(4) The quantum chemical calculation provides useful molecular properties, while it fails to allow good correlation of obtained descriptor with the experimental corrosion inhibition ranking due to no consideration of the interaction between inhibitor molecules and $\mathrm{Cu}$ substrate.

(5) The MD simulations shows that all of the investigated compounds adsorb tightly onto the Cu surface in a parallel state and that the $E_{\text {binding }}$ values agree well with the electrochemical results.

In summary, the halogeno-substitution can improve inhibitive performance of IA molecule and chloro-substituted molecule brings about the best ability, which is same as the phenomenon in neutral chloride solution. The superior inhibition efficiencies of the HIAs can be explained by the stronger interaction between HIA molecules and $\mathrm{Cu}$ surface and a larger coverage area for copper. Furthermore, this present study can help us to understand the effect of halogeno-substitution on the inhibition performance of IA molecule, and provide guidance to design novel related organics as effective corrosion inhibitors of copper.

\section{Conflicts of interest}

There are no conflicts to declare.

\section{Acknowledgements}

This research was supported by National Natural Science Foundation of China (No. 21676035, 21878029, 21706195), Sail plan of Guangdong, China (No. 2015YT02D025).

\section{References}

1 S. M. Sherif el, R. M. Erasmus and J. D. Comins, J. Colloid Interface Sci., 2007, 311, 144-151.

2 H. Bi, G. T. Burstein, B. B. Rodriguez and G. Kawaley, Corros. Sci., 2016, 102, 510-516.

3 W. Liu, Q. Xu, J. Han, X. Chen and Y. Min, Corros. Sci., 2016, 110, 105-113.

4 N. D. Nam, V. Q. Thang, N. T. Hoai and P. V. Hien, Corros. Sci., 2016, 112, 451-461.

5 M. Bozorg, T. Shahrabi Farahani, J. Neshati, Z. Chaghazardi and G. Mohammadi Ziarani, Ind. Eng. Chem. Res., 2014, 53, 4295-4303.

6 C. Zhang, H. Duan and J. Zhao, Corros. Sci., 2016, 112, 160169. 
7 Y. Qiang, S. Zhang, B. Tan and S. Chen, Corros. Sci., 2018, 133, 6-16.

8 S. Mo, H. Q. Luo and N. B. Li, J. Colloid Interface Sci., 2017, 505, 929-939.

9 P. Kannan, T. S. Rao and N. Rajendran, J. Colloid Interface Sci., 2018, 512, 618-628.

10 Z. Zhang, F. Wang, Y. Liu, S. Wu, W. Li, W. Sun, D. Guo and J. Jiang, RSC Adv., 2018, 8, 20648-20654.

11 Z. Cheng, S. Mo, J. JIa, J. Feng, H. Q. Luo and N. B. Li, RSC $A d v .$, 2016, 6, 15210-15219.

12 Y. Qiang, S. Zhang, S. Xu and L. Yin, $R S C A d v ., 2015,5$, 63866-63873.

13 G. Moretti and F. Guidi, Corros. Sci., 2002, 44, 1995-2011.

14 E. Stupnisek-Lisac, A. Gazivoda and M. Madzarac, Electrochim. Acta, 2002, 47, 4189-4194.

15 M. A. Amin and K. F. Khaled, Corros. Sci., 2010, 52, 11941204.

16 Y. Qiang, S. Zhang, L. Guo, X. Zheng, B. Xiang and S. Chen, Corros. Sci., 2017, 119, 68-78.

17 Z. Z. Tasic, M. M. Antonijevic, M. B. Petrovic Mihajlovic and M. B. Radovanovic, J. Mol. Liq., 2016, 219, 463-473.

18 G. Quartarone, M. Battilana, L. Bonaldo and T. Tortato, Corros. Sci., 2008, 50, 3467-3474.

19 A. Döner, A. O. Yüce and G. Kardaş, Ind. Eng. Chem. Res., 2013, 52, 9709-9718.

20 K. F. Khaled, Corros. Sci., 2010, 52, 3225-3234.

21 I. B. Obot, D. D. Macdonald and Z. M. Gasem, Corros. Sci., 2015, 99, 1-30.

22 Y. Wang and Y. Zuo, Corros. Sci., 2017, 118, 24-30.

23 G. L. F. Mendonça, S. N. Costa, V. N. Freire, P. N. S. Casciano, A. N. Correia and P. d. Lima-Neto, Corros. Sci., 2017, 115, 4155.

24 A. Ehsani, M. G. Mahjani, M. Hosseini, R. Safari, R. Moshrefi and H. Mohammad Shiri, J. Colloid Interface Sci., 2017, 490, 444-451.

25 Y. Qiang, S. Fu, S. Zhang, S. Chen and X. Zou, Corros. Sci., 2018, 140, 111-121.

26 L. Guo, S. Zhu, S. Zhang, Q. He and W. Li, Corros. Sci., 2014, 87, 366-375.

27 D. Wang, D. Yang, D. Zhang, K. Li, L. Gao and T. Lin, Appl. Surf. Sci., 2015, 357, 2176-2183.

28 N. Kovačević, I. Milošev and A. Kokalj, Corros. Sci., 2015, 98, 457-470.

29 Y. Qiang, S. Zhang, S. Xu and W. Li, J. Colloid Interface Sci., 2016, 472, 52-59.
30 Y. Qiang, S. Zhang, S. Yan, X. Zou and S. Chen, Corros. Sci., 2017, 126, 295-304.

31 D. Zhang, Y. Tang, S. Qi, D. Dong, H. Cang and G. Lu, Corros. Sci., 2016, 102, 517-522.

32 K. Azzaoui, E. Mejdoubi, S. Jodeh, A. Lamhamdi, E. Rodriguez-Castellón, M. Algarra, A. Zarrouk, A. Errich, R. Salghi and H. Lgaz, Corros. Sci., 2017, 129, 70-81.

33 H. Tian, W. Li, B. Hou and D. Wang, Corros. Sci., 2017, 117, 43-58.

34 X. Luo, X. Pan, S. Yuan, S. Du, C. Zhang and Y. Liu, Corros. Sci., 2017, 125, 139-151.

35 Z. Wang, Y. Gong, C. Jing, H. Huang, H. Li, S. Zhang and F. Gao, Corros. Sci., 2016, 113, 64-77.

36 Z. Tao, W. He, S. Wang and G. Zhou, Ind. Eng. Chem. Res., 2013, 52, 17891-17899.

37 Y. Yu, D. Zhang, H. Zeng, B. Xie, L. Gao and T. Lin, Appl. Surf. Sci., 2015, 355, 1229-1237.

38 K. F. Khaled, Electrochim. Acta, 2009, 54, 4345-4352.

39 Y. Hao, L. A. Sani, T. Ge and Q. Fang, Corros. Sci., 2017, 123, 158-169.

40 G. Khan, W. J. Basirun, S. N. Kazi, P. Ahmed, L. Magaji, S. M. Ahmed, G. M. Khan and M. A. Rehman, J. Colloid Interface Sci., 2017, 502, 134-145.

41 Y. Qiang, L. Guo, S. Zhang, W. Li, S. Yu and J. Tan, Sci. Rep., 2016, 6, 33305.

42 S. Ogata, N. Kobayashi, T. Kitagawa, S. Shima, A. Fukunaga, C. Takatoh and T. Fukuma, Corros. Sci., 2016, 105, 177-182.

43 A. Pourghasemi Hanza, R. Naderi, E. Kowsari and M. Sayebani, Corros. Sci., 2016, 107, 96-106.

44 Q. Ma, S. Qi, X. He, Y. Tang and G. Lu, Corros. Sci., 2017, 129, 91-101.

45 L. Cai, X. Feng, X. Hua, X. He, Y. Long and G. Chen, RSC Adv., 2016, 6, 68351-68356.

46 M. A. Bedair, M. M. B. El-Sabbah, A. S. Fouda and H. M. Elaryian, Corros. Sci., 2017, 128, 54-72.

47 H. M. Abd El-Lateef, M. A. Abo-Riya and A. H. Tantawy, Corros. Sci., 2016, 108, 94-110.

48 R. Kumar, O. S. Yadav and G. Singh, J. Mol. Liq., 2017, 237, 413-427.

49 L. Feng, H. Yang, X. Cui, D. Chen and G. Li, RSC Adv., 2018, 8, 6507-6518.

50 A. Dutta, S. K. Saha, U. Adhikari, P. Banerjee and D. Sukul, Corros. Sci., 2017, 123, 256-266.

51 Y. Meng, W. Ning, B. Xu, W. Yang, K. Zhang, Y. Chen, L. Li, X. Liu, J. Zheng and Y. Zhang, RSC Adv., 2017, 7, 4301443029. 